\title{
Expression and localization of FOXO1 in non-small cell lung cancer
}

\author{
TAKAYO MAEKAWA ${ }^{1}$, YOSHIMASA MANIWA ${ }^{1}$, TAKEFUMI DOI $^{3}$, WATARU NISHIO ${ }^{1}$, \\ MASAHIRO YOSHIMURA ${ }^{1,5}$, CHIHO OHBAYASHI ${ }^{4,6}$, YOSHITAKE HAYASHI ${ }^{3}$ and YUTAKA OKITA ${ }^{2}$
}

\author{
Divisions of ${ }^{1}$ Thoracic Surgery, ${ }^{2}$ Cardiovascular Surgery, ${ }^{3}$ Molecular Medicine and Medical Genetics, \\ International Center for Medical Research and Treatment; ${ }^{4}$ Department of Pathology, Kobe University Hospital, Kobe \\ University Graduate School of Medicine; Departments of ${ }^{5}$ Respiratory Surgery, ${ }^{6}$ Pathology, Hyogo Cancer Center, Japan
}

Received November 19, 2008; Accepted February 4, 2009

\section{DOI: $10.3892 /$ or_ 00000406}

\begin{abstract}
FOXO1 (FKHR), one of the forkhead family of transcription factors, is involved in proapoptotic signaling. Phosphorylated Akt leads to the inhibition of FOXO1 activity by direct phosphorylation and cytoplasmic sequestration. We investigated the localization of FOXO1 in non-small cell lung cancer (NSCLC) cell lines grown in a variety of media. FOXO1 expression in tissue microarrays of clinical NSCLC samples was analyzed immunohistochemically. Significant correlations between these data were assessed in order to discern the relationship between Akt and apoptosis. While FOXO1 localized to the nucleus in cell lines cultured without FBS, FOXO1 translocated from the nucleus to the cytoplasm in cell lines cultured with fetal bovine serum (FBS). Inhibition of Akt using RNA interference resulted in the accumulation of FOXO1 in A549 and EBC1 cell nuclei, and in accelerated apoptosis induction. In NSCLC cells, where FOXO1 was present in the cytoplasm constitutively, inhibition of Akt led to FOXO1 translocation to the nucleus and initiation of apoptosis. Using immunohistochemistry and immunofluorescence to examine the expression of FOXO1 in 185 surgically resected, paraffin-embedded stage I-IV tumor samples, we observed a significant correspondence between positive staining for FOXO1 in early stage tumors and in tumors without nodal involve-ment; in contrast, no significant relationship was observed between FOXO1 expression and tumor status, lymphatic invasion, or venous
\end{abstract}

Correspondence to: Dr Yoshimasa Maniwa, Division of Thoracic Surgery, Kobe University Graduate School of Medicine, Kobe, Japan

E-mail:maniwa@med.kobe-u.ac.jp

Abbreviations: NSCLC, non-small cell lung cancer; BAC, bronchio-alveolar carcinoma; FBS, fetal bovine serum; PI3K, phosphatidyl-inositol 3-kinase

Key words: FOXO1, Akt/PKB, non-small cell lung cancer, apoptosis invasion. Moreover, induction of apoptosis was vigorous in NSCLC cells expressing FOXO1. These results suggest that FOXO1 expression is a favorable prognostic factor in NSCLC.

\section{Introduction}

Forkhead transcription factors - FOXO1 (FKHR), FOXO3a (FKHRL1) and FOXO4 (AFX) - are mammalian homologues of DAF16, which is a critical regulator of the life span in Caenorhabditis elegans (1-3). Forkhead transcription factors are also known to be key regulators in glucose homeostasis (4), energy metabolism $(5,6)$, cell cycle arrest $(7,8)$, resistance to oxidative stress $(9,10)$, and apoptosis $(11,12)$. FOXO factors play a pivotal role in tumor suppression by inducing growth arrest and apoptosis (12), and are important targets in insulin and growth factor signaling pathways involving glucose-6-phosphatase (G6P), insulinlike-growth factor-1 (IGF-1), phosphatidyl-inositol 3-kinase (PI3K), and Akt/PKB (protein kinase B) $(13,14)$. The insulin response system and downstream components, such as PI3K, are activated by the presence of insulin, growth factors, and binding of certain cytokines to their receptors at the cell membrane. These events promote activation of Akt, which phosphorylates FOXO proteins $(13,15-17)$, resulting in their inactivation and sequestration in the cytoplasm $(12,13)$. FOXO1 phosphorylation not only results in nuclear exclusion, but also promotes ubiquitin-mediated protein degradation $(14,18,19)$.

Forkhead proteins remain unphosphorylated when Akt activity is inhibited by PTEN or PI3K inhibitors (15). During PI3K/Akt pathway inhibition, FOXOs localize to the nucleus and exist in an active state, where they promote cell death by inducing the expression of genes critical for apoptosis, including the Fas ligand, TRADD, TRAIL, Bim, $p 27^{K I P I}(12,13,16,20-23)$. Additional pathways that inhibit FOXO functions have also been reported $(24,25)$.

Akt promotes cell proliferation and survival by regulating metabolic homeostasis and transcription factors that control the expression of pro- and anti-apoptotic genes $(13,14,19,21,26)$. Activation of Akt at the plasma membrane is followed by its translocation to both the cytoplasm 


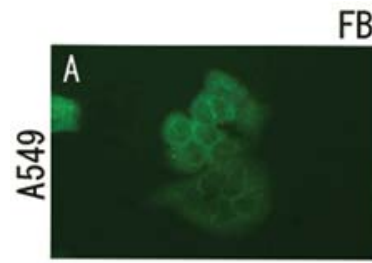

FBS $(+)$
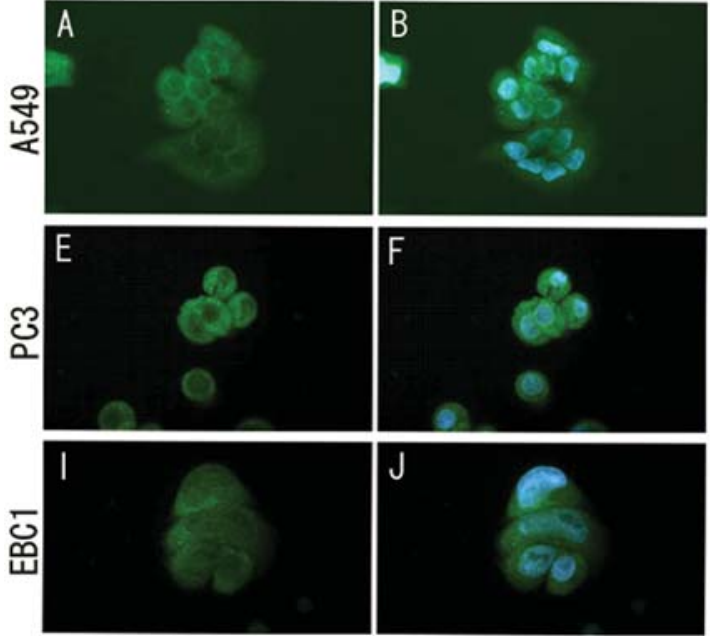

FITC (F0X01)
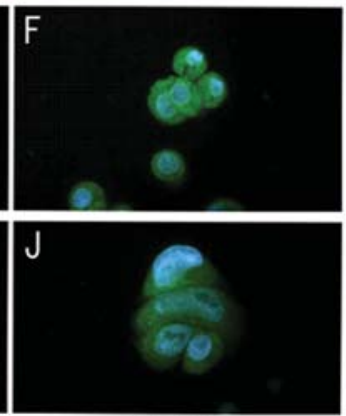

Merge

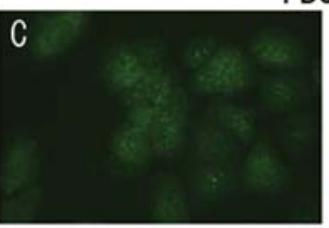

FBS $(-)$
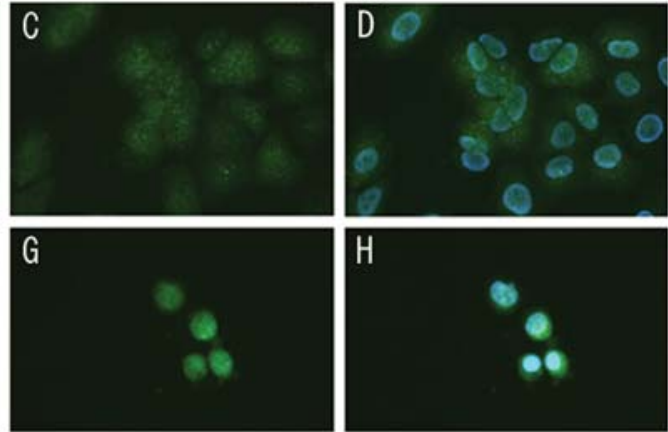

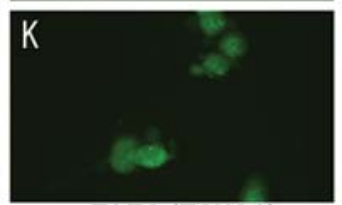

FITC(FOX01)

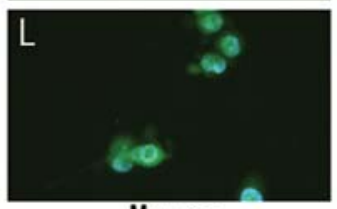

Merge

Figure 1. Localization of FOXO1 in the NSCLC cell lines. Three types of cells were cultured with or without FBS. In the presence of FBS, FOXO1 was localized in the cytoplasm for all the cell lines (A, B, E, F, I and J). In contrast, FOXO1 was localized in the nucleus in the absence of FBS (C, D, G, H, K and L).

and the nucleus (21). Activation can also occur through mutation-induced loss of the tumor suppressor PTEN, or by activation of an oncogenic receptor tyrosine kinase $(21,25,26)$. Although Akt negatively regulates FOXO1 by direct phosphorylation, it also positively regulates at least two other transcription factors, including $\mathrm{NF}-\kappa \mathrm{B}$ and CREB (21). Several previous studies have investigated PI3K, Akt and insulin-like growth factor binding protein-3 (IGFBP-3) in NSCLC cells (27-32), a line in which Akt appears to be constitutively active $(27,29)$. Balsara et al suggested that increased Akt 2 activity could play a role in local invasion during early stages of tumorigenesis (27). Akt has also been reported to play a role in resistance to chemotherapy and irradiation (29). A high level of phosphorylated Akt protein is a favorable prognostic indicator in NSCLC, independent of cancer stage (28).

Although clinical features of FOXO1 expression have rarely been reported, the findings described above suggest that the Akt/FOXO1 pathway could influence biological features of NSCLC. In this study, we examined the localization of FOXO1 in NSCLC cell lines grown under several conditions. We also analyzed FOXO1 expression in a tissue microarray of clinical NSCLC samples, and have examined relationships between FOXO1 expression, Akt and apoptosis.

\section{Materials and methods}

Cell lines. All non-small cell lung cancer (NSCLC) cell lines were provided by the RIKEN BioResource Center. To evaluate the localization of FOXO1, the following three NSCLC cell lines were used: A549 (bronchio-alveolar carcinoma; BAC), PC3 (adenocarcinoma), and EBC1 (squamous carcinoma). These cell lines were cultured in RPMI1640 medium (Life Technologies, Inc., Rockville, MD) containing $10 \%$ fetal bovine serum (FBS), $100 \mathrm{U} / \mathrm{ml}$ of penicillin, and $100 \mathrm{U} / \mathrm{ml}$ of streptomycin in a humidified atmosphere containing $5 \% \mathrm{CO}_{2}$ at $37^{\circ} \mathrm{C}$.

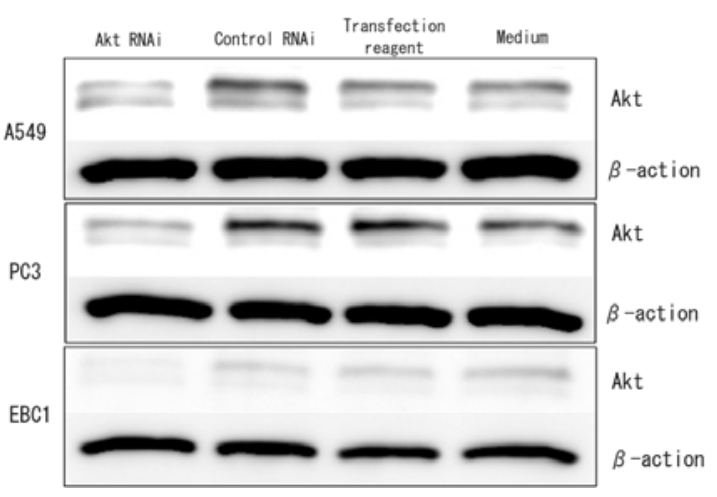

Figure 2. Akt expression in NSCLC cell lines after knock-down using RNA interference. After knockdown using RNA interference (Akt-RNAi), Akt expression was remarkably reduced in all cell lines relative to controls (control RNAi, transfection reagent, and only the medium solution). B-actin was used as a loading control.

Paraffin sections and tissue microarray. All primary lung cancer specimens were derived from patients who underwent surgery at Kobe University Hospital between April 2001 and December 2005. Excluding squamous cell carcinomas, we examined 185 samples of adenocarcinoma. Baseline characteristics of the samples are presented in Table I. Tissue microarrays were constructed by harvesting 1-mm tissue cores from paraffin-embedded samples of the main lesion. The tissue core punches were arrayed in $15 \times 15 \mathrm{~mm}^{2}$ paraffin blocks. The Ethics Committee of Kobe University Hospital approved this study.

Immunohistochemistry. FOXO1 expression in paraffin sections and tissue microarray samples was analyzed immunohistochemically. FKHR primary antibody (antihuman FOXO1 rabbit polyclonal antibody, no.9462; Cell Signaling Technology, Inc., Danvers, MA), diluted to 1:100 in blocking solution, was added to the tissue sections and 

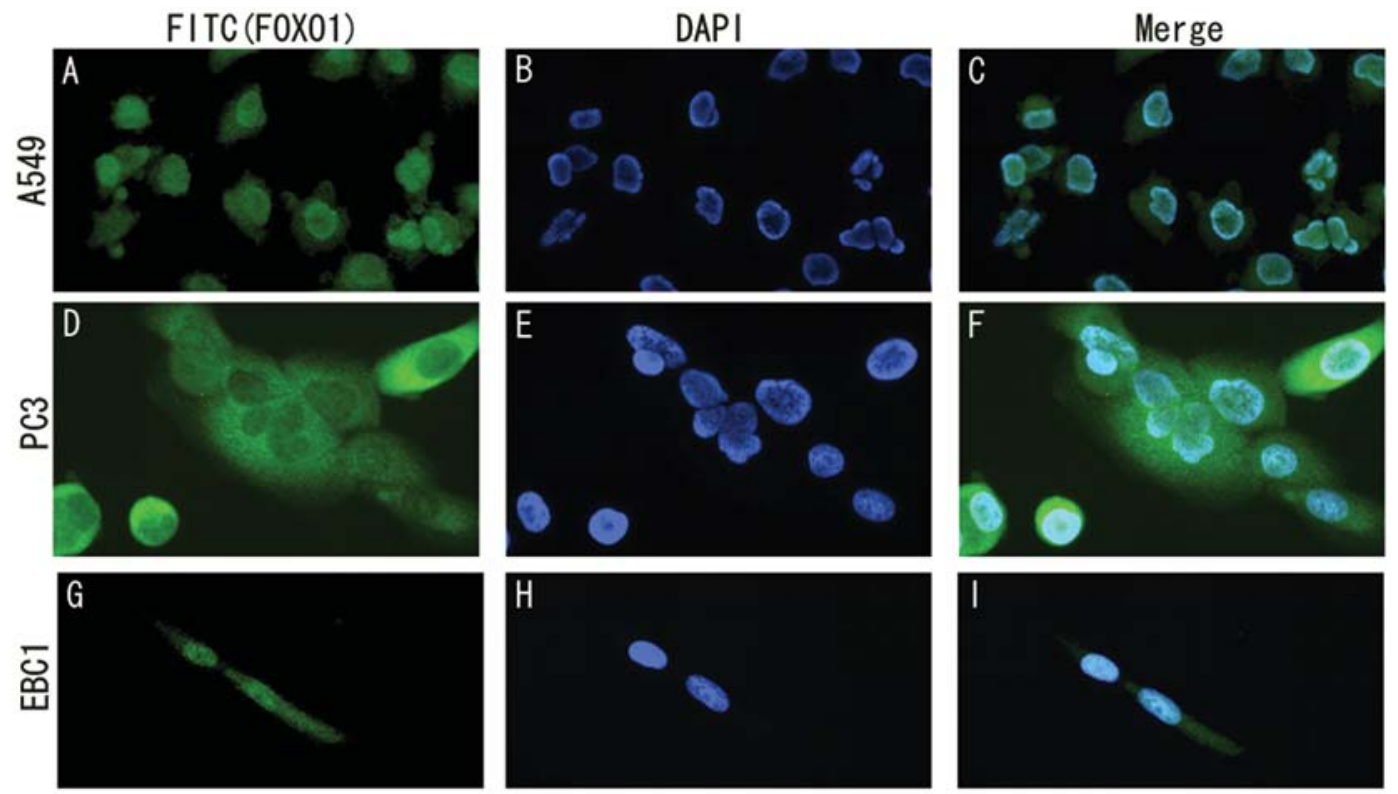

Figure 3. Localization of FOXO1 in Akt knock-down cell lines. Strong accumulation of FOXO1 in the nucleus was observed in A549 and EBC1 cell lines (A, C, G and I). In PC3 cells, FOXO1 localized to the cytoplasm (D and F).
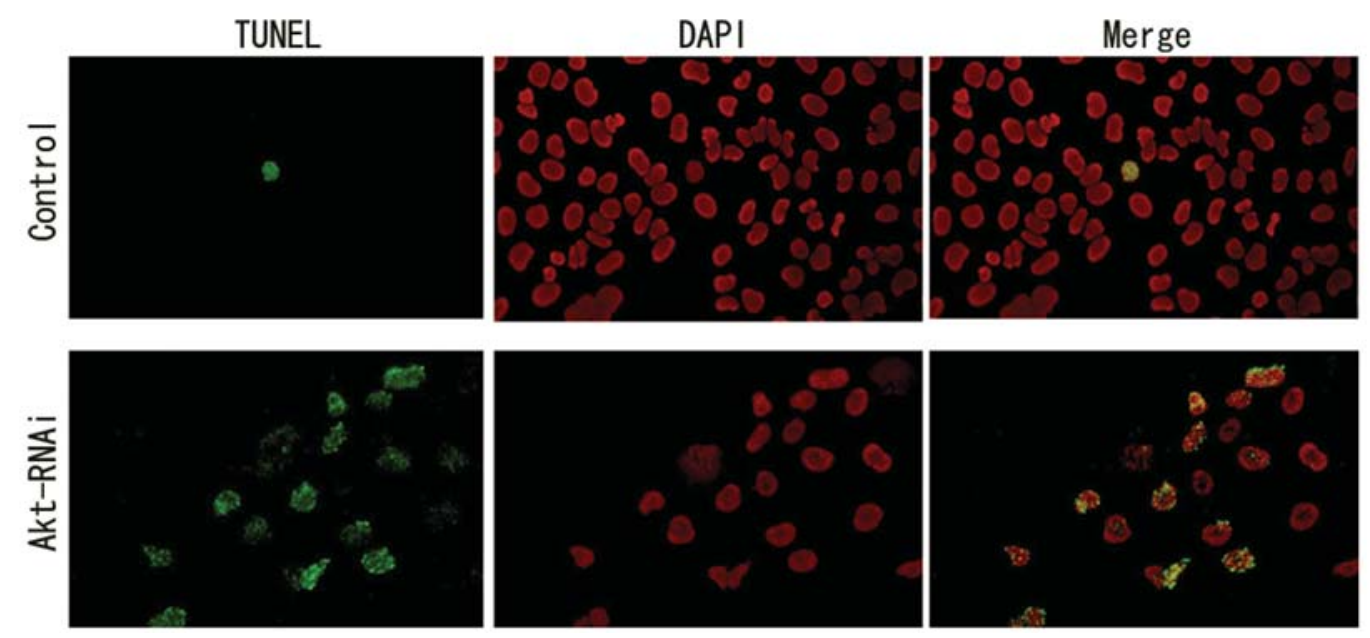

Figure 4. Detection of apoptosis in NSCLC cell lines. Using TUNEL staining, apoptosis was rarely detected in controls (A, B and C). In cells subjected to Akt-RNAi, the induction of apoptosis was remarkably accelerated (D, E and F).

incubated overnight at $4^{\circ} \mathrm{C}$. Antigen-antibody complexes were detected by the avidin-biotin peroxidase method using ABC (Vectastain Elite ABC Kit, Vector Laboratories, Inc., Burlingame, CA) and DAB (Dako EnVision ${ }^{\mathrm{TM}} / \mathrm{HRP}$, Dako, Japan) reagents. Sections were counterstained with hematoxylin.

Immunofluorescence. Immunofluorescence was performed on cell lines and tissue microarray samples. Cells were washed briefly in 1x PBS and fixed in 2\% paraformaldehyde/ $1 \mathrm{x}$ PBS for $15 \mathrm{~min}$ at $37^{\circ} \mathrm{C}$. Cells were then permeabilized by incubating with $0.5 \%$ Triton X/PBS for $15 \mathrm{~min}$ at room temperature. After washing, cells were incubated in blocking buffer $\left(1 \%\right.$ of BSA/PBS) for $30 \mathrm{~min}$ at $37^{\circ} \mathrm{C}$. Both cell lines and tissue microarray samples were incubated with antihuman FOXO1 rabbit polyclonal FKHR antibody (no. 9462; Cell Signaling Technology, Inc.) (1:50) overnight at $4^{\circ} \mathrm{C}$.
After washing, cells were incubated with FITC-labeled antirabbit Ig donkey whole antibody (Amersham Biosciences Corp., Piscataway, NJ) for $30 \mathrm{~min}$ at room temperature, and nuclei were then counter-stained with DAPI (Antifade solution; Chemicon International, Inc., Temecula, CA).

Akt knock-down. A549, PC3 and EBC1 cells were cultured with an Akt small interfering ribonucleic acid (siRNA) (SI00299145; Qiagen, Inc., Tokyo, Japan), non-specific siRNA (no. 1022076; Qiagen, Inc.), and HiPerFect Transfection Reagent (no. 301705; Qiagen, Inc). The culture solution was changed after $6 \mathrm{~h}$, after which the cell lines were cultured for an additional $42 \mathrm{~h}$.

Western blot analysis. Transfected cells were dissolved in $100 \mu 1$ Laemmli sample buffer. Equal amounts of cell lysate proteins were resolved on sodium dodecyl sulfate-poly- 
Table I. Baseline characteristics of the 185 NSCLC samples.

\begin{tabular}{|c|c|}
\hline Total (N) & 185 \\
\hline Median Age ( \pm SD) & $68.14( \pm 8.67)$ \\
\hline \multicolumn{2}{|l|}{ Gender } \\
\hline Female & 75 \\
\hline Male & 110 \\
\hline \multicolumn{2}{|l|}{ Histological subtype } \\
\hline Mixed subtype & 79 \\
\hline Papillary & 42 \\
\hline Bronchio-alveolar & 31 \\
\hline Poorly & 12 \\
\hline Acinar & 6 \\
\hline Solid & 5 \\
\hline Tubular & 4 \\
\hline Others & 6 \\
\hline \multicolumn{2}{|l|}{ Tumor status } \\
\hline $\mathrm{T} 1$ & 107 \\
\hline $\mathrm{T} 2$ & 61 \\
\hline $\mathrm{T} 3$ & 7 \\
\hline $\mathrm{T} 4$ & 10 \\
\hline \multicolumn{2}{|l|}{ Nodal status } \\
\hline No & 139 \\
\hline N1 & 21 \\
\hline$\geq \mathrm{N} 2$ & 25 \\
\hline \multicolumn{2}{|l|}{ Pathological stage } \\
\hline I & 129 \\
\hline II & 22 \\
\hline III & 22 \\
\hline IV & 4 \\
\hline
\end{tabular}

acrylamide gels (SDS-PAGE) and then transferred to nitrocellulose. After washing and blocking, the membrane was incubated with primary antibody (anti-Akt antibody, no.9272, diluted to 1:1000; Cell Signaling Technology, Inc.) for $1 \mathrm{~h}$ at room temperature. After washing, the membrane was incubated with secondary antibody (peroxidase-labeled anti-rabbit Ig donkey whole antibody; Amersham Biosciences Corp.). Reactive proteins were visualized using enhanced chemiluminescence detection (Amersham Biosciences Corp.) and analyzed using the lumino-image analyzer LAS-3000 (Fuji Film Inc., Tokyo, Japan). The same membrane was treated with anti- $\beta$-actin antibody (no. AB6276; Abcam, Cambridge, UK) for use as a loading control.

TUNEL staining. The In Situ Cell Death Detection kit, Fluorescein (POD no. 1684817; Roche Diagnostics KK, Indianapolis, IN) was used for terminal deoxynucleotidyl transferase (TdT)-mediated 2-deoxyuridine 5-triphosphate (dUTP) nick-end labeling (TUNEL) to detect apoptotic figures in cultured cells and in tissue microarrays. The kit was used according to the manufacturer's instructions.
Table II. Clinicopathological aspects of the tissue microarray.

\begin{tabular}{|c|c|c|c|c|}
\hline & 0 & $1+$ & $2+^{a}$ & $\mathrm{n}$ \\
\hline Total & 36 & 78 & 71 & 185 \\
\hline \multicolumn{5}{|l|}{ Histological subtype } \\
\hline Mixed subtype & 13 & 40 & 26 & 79 \\
\hline Papillary & 5 & 17 & 20 & 42 \\
\hline Bronchio-alveolar & 4 & 12 & 15 & 31 \\
\hline Poorly & 5 & 3 & 4 & 12 \\
\hline Acinar & 2 & 4 & 0 & 6 \\
\hline Solid & 3 & 0 & 2 & 5 \\
\hline Tubular & 1 & 1 & 2 & 4 \\
\hline Mucinous & 0 & 1 & 2 & 3 \\
\hline Signet cell & 2 & 0 & 0 & 2 \\
\hline Adenosquamous & 1 & 0 & 0 & 1 \\
\hline \multicolumn{5}{|l|}{ Tumor status ${ }^{\mathrm{b}}$} \\
\hline $\mathrm{T} 1$ & 19 & 41 & 47 & 107 \\
\hline $\mathrm{T} 2$ & 12 & 29 & 20 & 61 \\
\hline $\mathrm{T} 3$ & 4 & 3 & 0 & 7 \\
\hline $\mathrm{T} 4$ & 1 & 5 & 4 & 10 \\
\hline \multicolumn{5}{|l|}{ Nodal status ${ }^{\mathrm{c}}$} \\
\hline No & 22 & 63 & 54 & 139 \\
\hline$\geq \mathrm{N} 1$ & 14 & 15 & 17 & 46 \\
\hline \multicolumn{5}{|l|}{ Pathological stage } \\
\hline I & 18 & 59 & 52 & 129 \\
\hline II & 10 & 5 & 7 & 22 \\
\hline III & 8 & 10 & 12 & 30 \\
\hline IV & 0 & 4 & 0 & 4 \\
\hline \multicolumn{5}{|c|}{ Lymphatic invasion (ly) } \\
\hline Negative & 19 & 46 & 47 & 112 \\
\hline Positive & 17 & 32 & 24 & 73 \\
\hline \multicolumn{5}{|l|}{ Venous invasion (v) } \\
\hline Negative & 1 & 48 & 47 & 112 \\
\hline Positive & 19 & 3 & 24 & 73 \\
\hline \multicolumn{5}{|l|}{ TUNEL staining $^{\mathrm{d}}$} \\
\hline 0 & 13 & 40 & 32 & 85 \\
\hline$<10 \%$ & 13 & 33 & 36 & 82 \\
\hline$\geq 10 \%$ & 10 & 5 & 3 & 18 \\
\hline
\end{tabular}

${ }^{\text {aS }}$ Samples were categorized into one of three groups based on the degree of staining as follows: 0 , negative staining compared to the non-specific background staining; $2+$, strong positive staining compared to the background; $1+$, positive staining but weaker than $2+{ }^{\mathrm{b}, \mathrm{c}}$ According to the TNM classification of UICC. ${ }^{\mathrm{d}}$ The results of TUNEL staining were evaluated as follows: 0 , no accumulation was observed; $<10 \%$, positive staining present but $<10 \%$; $\geq 10 \%$, positive staining $\geq 10 \%$ observed.

Statistical analysis. All statistical calculations were performed using the $\chi^{2}$ test with Yates' correction. Differences were considered statistically significant at $\mathrm{P}<0.05$. 
(A)

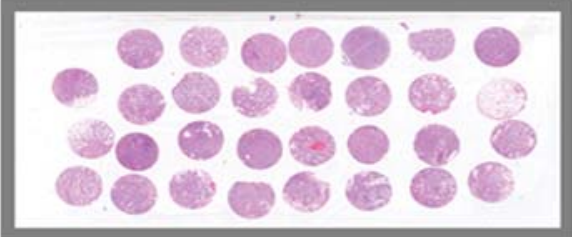

(B)

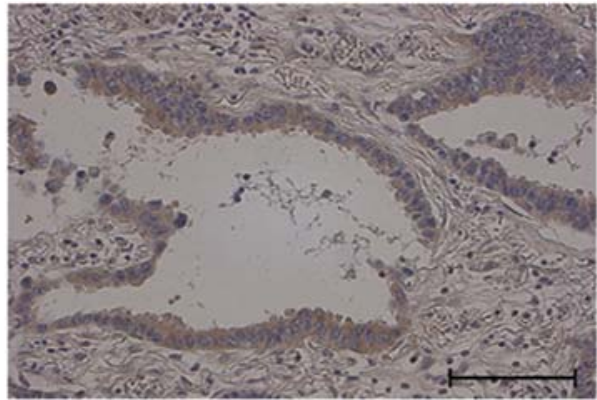

(C)

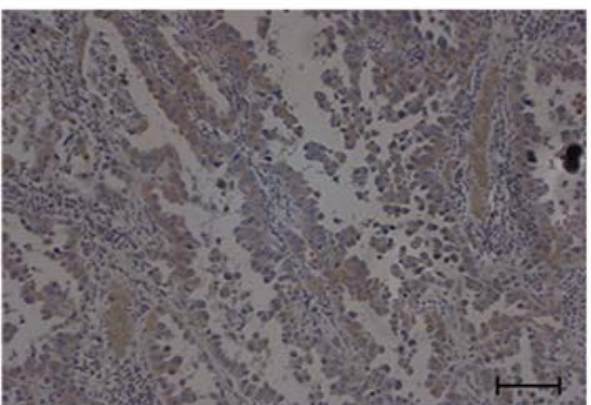

Figure 5. Tissue microarray and immunohistochemical analyses of clinical NSCLC samples. (A) A tissue microarray slide with H\&E staining. Each slide contains 30-32 samples. (B and C) Immunohistochemistry with FKHR (FOXO1) antibody.
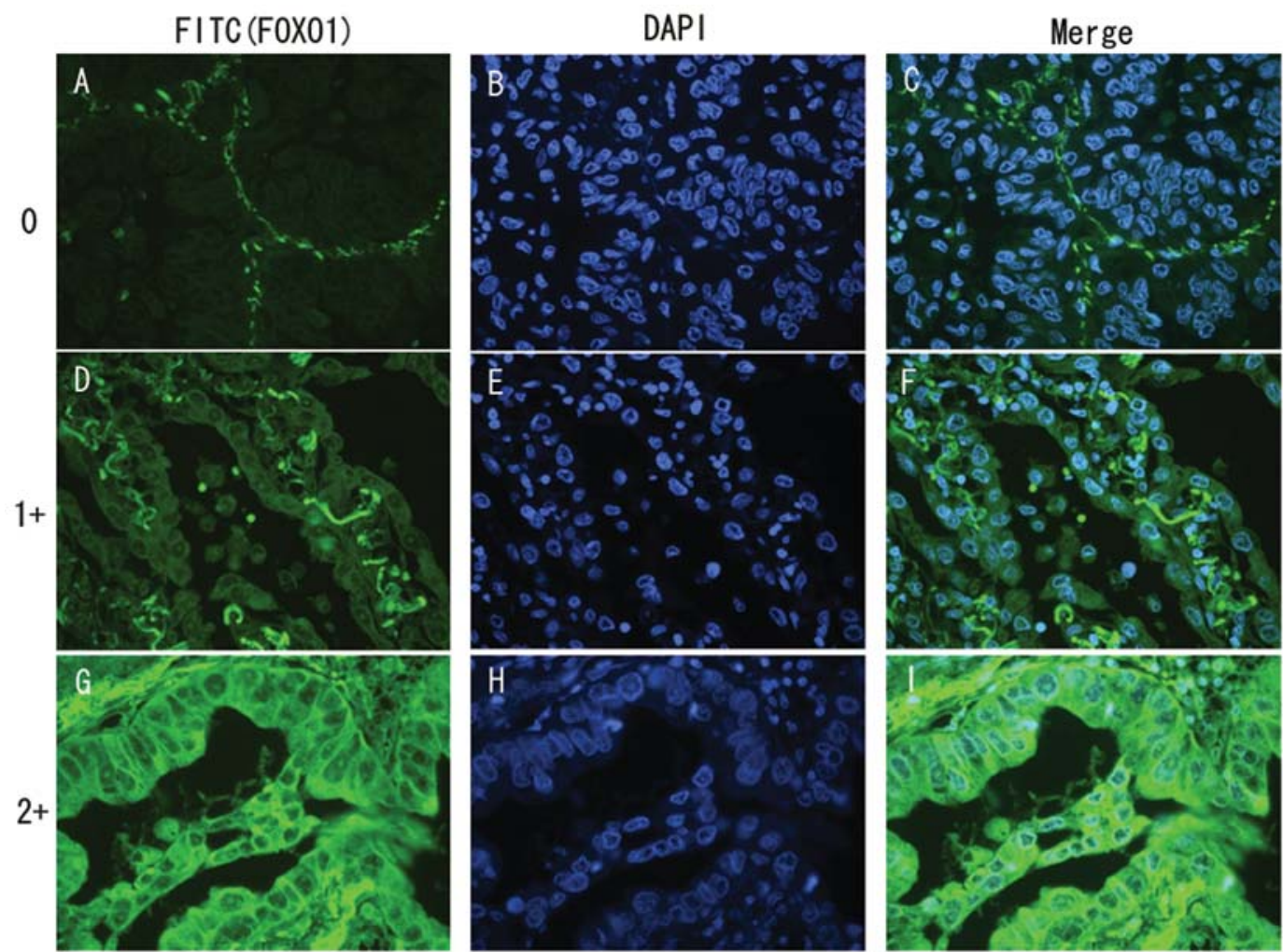

Figure 6. Immunofluorescence in tissue microarray. Samples were categorized into one of three groups based on degree of staining as follows: 0 , negative staining compared to the non-specific background staining (A, B and C); 2+, strong positive staining compared to the background (G, H and I); 1+, positive staining but weaker than $2+(\mathrm{D}, \mathrm{E}$ and $\mathrm{F})$.

\section{Results}

Localization of FOXO1 in NSCLC cell lines with or without FBS. Three cell lines (A549, PC3 and EBC1) cultured with or without FBS (Fig. 1) were analyzed immunohistochemically. All cell lines cultured with FBS demonstrated FOXO1 localization to the cytoplasm. In contrast, FOXO1 was localized within the nucleus in cell lines cultured without FBS.
Localization of FOXO1 in Akt knocked-down NSCLC cell lines. To determine whether the change of FOXO1 localization in NSCLC cell lines was due to Akt activation, we used RNA-interference (RNAi) to suppress Akt expression. Western blot analysis of RNAi transfected cells confirmed that Akt expression was remarkably reduced in all cell lines (Fig. 2).

Immunofluorescence was used to visualize the localization of FOXO1 in Akt knock-down NSCLC cells. While 
Table III. Correlation between FOXO1 staining and clinicopathological characteristics using the $\chi^{2}$ test.

\begin{tabular}{|c|c|c|c|}
\hline & Negative & Positive $^{a}$ & p-value \\
\hline \multicolumn{4}{|c|}{ Tumor status } \\
\hline $\mathrm{T} 1$ & 19 & 88 & NS \\
\hline$\geq \mathrm{T} 2$ & 17 & 61 & \\
\hline \multicolumn{4}{|c|}{ Nodal status } \\
\hline NO & 22 & 117 & 0.050 \\
\hline$\geq \mathrm{N} 1$ & 14 & 32 & \\
\hline \multicolumn{4}{|c|}{ Pathological stage } \\
\hline I & 18 & 111 & 0.008 \\
\hline$\geq \mathrm{II}$ & 18 & 38 & \\
\hline \multicolumn{4}{|c|}{ Lymphatic invasion (ly) } \\
\hline Negative & 19 & 93 & NS \\
\hline Positive & 17 & 56 & \\
\hline \multicolumn{4}{|c|}{ Venous invasion (v) } \\
\hline Negative & 17 & 95 & NS \\
\hline Positive & 19 & 54 & \\
\hline \multicolumn{4}{|c|}{ TUNEL staining } \\
\hline$<10 \%$ b & 26 & 141 & 0.0002 \\
\hline$\geq 10 \%$ & 10 & 8 & \\
\hline
\end{tabular}

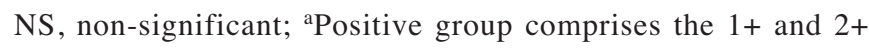
groups; ${ }^{b}<10 \%$ comprises both the negative and $<10 \%$ staining groups.

strong FOXO1 nuclear accumulation was detected in A549 and EBC1 cells, FOXO1 was excluded from the nucleus and present in the cytoplasm in PC3 cells (Fig. 3).

Detection of apoptosis in NSCLC cell lines. We used TUNEL staining in cells knocked down by RNAi to assess correlations between FOXO1 localization and apoptosis induction. Whereas apoptosis of control cells was infrequent, cells transfected with Akt-RNAi showed a significant apoptotic induction (Fig. 4).

Localization of FOXO1 and detection of apoptosis in clinical NSCLC samples. Anti-FOXO1 immunohistochemistry of paraffin-embedded sections and immunofluorescence of tissue microarray were also performed. Fig. 5 illustrates a hematoxylin and eosin (H\&E)-stained slide of one tissue microarray sample as well as positive immunostaining of paraffin sections. Baseline characteristics of 185 samples are described in Table I. Based on the degree of staining, we categorized samples into three groups as follows: 0 indicates negative staining relative to the non-specific background stain; $2+$ indicates strong staining relative to the background; $1+$ indicates intermediate, positive staining (Fig. 6). Table II describes the clinicopathological features of each group of tissue microarray samples. Among the three groups combined, $80.6 \%$ of all samples showed FOXO1 staining, with 36 FOXO1-positive samples $(19.4 \%)$ in group $0,78(42.2 \%)$ samples in group $1+$, and $71(38.4 \%)$ samples in group $2+$.
We also examined the relationship between the FOXO1 staining and clinicopathological factors (Table III). There was a significant positive correlation between FOXO1positive staining in samples without nodal involvement and those at an early pathological stage. In contrast, no significant correlation was observed between FOXO1-positive staining and tumor status or lymphatic and venous invasion. Among cell populations with some apoptotic induction, we observed a significant positive correlation between TUNEL-negative staining or $<10 \%$ staining and positive FOXO1 immunostaining.

There was consistency between immunofluorescence and immunohistochemistry results, in that similar staining patterns were observed in cells viewed with these techniques. FOXO1 expression was seen in both the cytoplasm and nucleus, although it was mainly detected in the cytoplasm; strict nuclear localization was detected infrequently in clinical tissue microarray samples. Although not statistically significant, we noted an interesting tendency for more frequent and intense FOXO1 staining in histologically welldifferentiated tumors.

\section{Discussion}

When NSCLC cell lines were cultured in media deprived of growth factors (without FBS), FOXO1 localized almost exclusively to the nucleus. In contrast, cells cultured with FBS showed FOXO1 excluded from the nucleus and localized to the cytoplasm. The Akt pathway is positively activated by growth factors; thus, growth factor deprivation results in pathway inhibition (13) and subsequent induction of FOXO1 translocation. With the exception of PC3 cells, suppression of Akt expression in NSCLC cells using RNAi also resulted in FOXO1 nuclear localization. Similar to a previous study by Brunet et al on FOXO3a (13), our study confirms that Akt activity in NSCLC cells is necessary for FOXO1 cytoplasmic sequestration. In PC3 cells, we hypothesize that other proteins, such as GSK (also known as the serum- and glucocorticoid-regulated kinase) (21), may also play an important role in the FOXO1 phosphorylation, or that mutations in the FOXO1 gene may prevent nuclear translocation.

Our experiments demonstrate that induction of apoptosis was accelerated in NSCLC cells transfected with inhibitory Akt RNA, whereas apoptosis was infrequent in the absence of Akt RNAi. According to Brunet et al (13), inactivation of the PI3K/Akt pathway results in an absence of FOXO1 phosphorylation, leading to accumulation of FOXO1 in the nucleus. It is in the nucleus where FOXO1 functions as a transcriptional activator of death genes, thereby inducing apoptosis.

FOXO1 immunohistochemistory and immunofluorescence in NSCLC paraffin-embedded samples revealed that $80.6 \%$ clinical samples showed FOXO1 positive staining. Balsara et al reported previously that there is a strong correlation between positive staining for phosphorylated FOXO1, detected in $68 \%$ of tumors, and Akt activation (27). In our clinical NSCLC samples, FOXO1 was largely present both in the cytoplasm and nucleus. In contrast, NSCLC cell lines showed constitutive expression of FOXO1 in the 
cytoplasm and translocation to the nucleus in response to inhibition of Akt. These results suggest that in clinical samples, FOXO1 localization is not necessarily a strict measure of its activity, and that FOXO1 expression may be a more critical factor than subcellular localization.

Among the clinicopathological factors examined in clinical NSCLC samples, we observed a significant positive correlation between FOXO1-positive staining and an absence of nodal involvement and an early pathological stage. In contrast, no significant correlations were observed with regard to FOXO1-positive staining and histological subtype, tumor status, lymphatic invasion and venous invasion.

Balsara et al previously reported frequent activation of the Akt pathway in primary NSCLCs, and they observed no obvious correlation between expression of phosphorylated Akt and tumor stage (27). Shah et al also reported high levels of phosphorylated Akt protein as a favorable prognostic factor in NSCLC, independent of stage (28). In contrast, another study noted an inverse correlation between cytoplasmic FOXO3a and survival rate in patients with breast cancer (19). Expression of the anti-apoptotic protein Bax inhibitor-1 gene in the BAC component of adenocarcinoma has also been noted to occur frequently in patients without vascular or pleural invasions, and with well-differentiated tumors (33). Our data indicating that FOXO1 expression may be related to early pathological stage show some similarities with these previous studies.

In NSCLC clinical samples, negative or $<10 \%$ TUNEL staining also correlated significantly to FOXO1 positive staining. Lee et al reported that IGFBP-3, a potent inhibitor of PI3K/Akt and MAPK signaling pathways, induces apoptosis in NSCLC cells (34). In another study, IGFBP-3 overexpression also induced apoptosis in NSCLC cells; however, Ras-mediated signaling pathways could promote resistance to IGFBP-3 apoptotic activity in NSCLC cells (31). The frequent decrease in IGFBP-3 expression in lung cancers correlates strongly with poor prognosis for patients with stage I NSCLC (32). Based on these results, it appears that the PI3K/Akt signaling pathway is normally activated and induces inactivation of FOXO1 to inhibit apoptosis in NSCLC cells. Thus, inhibition of the Akt pathway may be effective in tumor suppression.

In conclusion, in NSCLC cells, inhibition of Akt expression and a resulting change in FOXO1 localization lead to an acceleration in apoptosis. These data indicate that the Akt/FOXO1 pathway regulates apoptosis and contributes to tumor progression. In clinical NSCLC samples, expression of FOXO1 is related to early cancer status, suggesting that FOXO1 expression may be an appropriate marker for predicting prognosis. Further investigations of the signaling mechanism by which FOXO1 prevents tumor cell survival or resistance to apoptosis will be necessary. Ultimately, understanding inhibition of the Akt/FOXO1 signaling pathway may contribute to novel molecular targeted treatment strategies for NSCLC.

\section{References}

1. Furuyama T, Kitayama K, Shimoda Y, et al: Abnormal angiogenesis in Foxo1 (Fkhr)-deficient mice. J Biol Chem 279: 34741-34749, 2004.
2. Furuyama T, Nakazawa T, Nakano I, et al: Identification of the differential distribution patterns of mRNAs and consensus binding sequences for mouse DAF-16 homologues. Biochem J 349: 629-634, 2000.

3. Katoh M and Katoh M: Human FOX gene family (Review). Int J Oncol 25: 1495-1500, 2004.

4. Nakae J, Biggs WH III, Kitamura T, et al: Regulation of insulin action and pancreatic beta-cell function by mutated alleles of the gene encoding forkhead transcription factor Foxo1. Nat Genet 2: 245-253, 2002.

5. Accili D and Arden KC: FoxOs at the crossroads of cellular metabolism, differentiation, and transformation. Cell 117: 421-21, 2004.

6. Furuyama T, Kitayama K, Yamashita H, et al: Forkhead transcription factor FOXO1 (FKHR)-dependent induction of PDK4 gene expression in skeletal muscle during energy deprivation. Biochem J 375: 365-371, 2003.

7. Ramaswamy S, Nakamura N, Sansal I, et al: A novel mechanism of gene regulation and tumor suppression by the transcription factor FKHR. Cancer Cell 2: 81-91, 2002.

8. Medema RH, Kops GJPL, Bos JL, et al: AFX-like Forkhead transcription factors mediate cell-cycle regulation by Ras and PKB through p27kip1. Nature 404: 782-787, 2000.

9. Kawamori D, Kaneto H, Nakatani Y, et al: The forkhead transcription factor Foxol bridges the JNK pathway and the transcription factor PDX-1 through its intracellular translocation. J Biol Chem 281: 1091-1098, 2006.

10. Tothova Z, Kollipara R, Huntly BJ, et al: FoxOs are critical mediators of hematopoietic stem cell resistance to physiologic oxidative stress. Cell 128: 325-339, 2007.

11. Tang ED, Nunez G, Barr FG, et al: Negative regulation of the Forkhead transcription factor FKHR by Akt. J Biol Chem 274: 16741-16746, 1999.

12. Huang H, Regan KM, Wang F, et al: Skp2 inhibits FOXO1 in tumor suppression through ubiquitin-mediated degradation. Proc Natl Acad Sci USA 102: 1649-1654, 1999.

13. Brunet A, Bonni A, Zigmond MJ, et al: Akt promotes cell survival by phosphorylating and inhibiting a Forkhead transcription factor. Cell 96: 857-868, 1999.

14. Matsuzaki H, Daitoku H, Hatta M, et al: Insulin-induced phosphorylation of FKHR (Foxo1) targets to proteasomal degradation. Proc Natl Acad Sci USA 100: 11285-11290, 2003.

15. Nakae J, Barr V and Accili D: Differential regulation of gene expression by insulin and IGF-1 receptors correlates with phosphorylation of a single amino acid residue in the forkhead transcription factor FKHR. EMBO J 19: 989-996, 2000.

16. Burgering BM and Medema RH: Decisions on life and death: FOXO Forkhead transcription factors are in command when PKB/Akt is off duty. J Leukoc Biol 6: 689-701, 2003.

17. Nakae J, Park BC and Accili D: Insulin stimulates phosphorylation of the Forkhead transcription factor FKHR on serine 253 through a Wortmannin-sensitive pathway. J Biol Chem 274: 15982-15985, 1999.

18. Zhang X, Gan L, Pan H, et al: Phosphorylation of serine 256 suppresses transactivation by FKHR (FOXO1) by multiple mechanisms. J Biol Chem 277: 45276-45284, 2002.

19. Hu MCT, Lee DF, Xia W, et al. IкB kinase promotes tumorigenesis through inhibition of Forkhead FOXO3a. Cell 117: 225-237, 2004.

20. Gilley J, Coffer PJ and Ham J: FOXO transcription factors directly activate bim gene expression and promote apoptosis in sympathetic neurons. J Cell Biol 162: 613-622, 2003.

21. Nicholson KM and Anderson NG: The protein kinase B/Akt signaling pathway in human malignancy. Cell Signal 14: 381-395, 2002.

22. Morris JB, Kenney B, Huynh H, et al: Regulation of the proapoptotic factor FOXO1 (FKHR) in cardiomyocytes by growth factors and $\alpha 1$-adrenergic agonists. Endocrinology 146: 4370-4376, 2005.

23. Huang H, Muddiman DC and Tindall DJ: Androgens negatively regulate Forkhead transcription factor FKHR (FOXO1) through a proteolytic mechanism in prostate cancer cells. J Biol Chem 279: 13866-13877, 2004.

24. Daly C, Wong V, Burova E, et al: Angiopoietin-1 modulates endothelial cell function and gene expression via the transcription factor FKHR (FOXO1). Genes Dev 18: 1060-1071, 2004.

25. Nakamura N, Ramaswamy S, Vazquez F, et al: Forkhead transcription factors are critical effectors of cell death and cell cycle arrest downstream of PTEN. Mol Cell Biol 20: 8969-8982, 2000 . 
26. Vivanco I and Sawyers CL: The phosphatidylinositol 3-kinaseAkt pathway in human cancer. Nat Rev Cancer 2: 489-501, 2002.

27. Balsara BR, Pei J, Mitsuuchi Y, et al: Frequent activation of AKT in non-small cell lung carcinomas and preneoplastic bronchial lesions. Carcinogenesis 25: 2053-2059, 2004

28. Shah A, Swain WA, Richardson D, et al: Phospho-Akt expression is associated with a favorable outcome in non-small cell lung cancer. Clin Cancer Res 11: 2930-2936, 2005.

29. Brognard J, Clark AS, Ni Y, et al: Akt/Protein kinase B is constitutively active in non-small cell lung cancer cells and promotes cellular survival and resistance to chemotherapy and radiation. Cancer Res 61: 3986-3997, 2001.

30. Denlinger CE, Rundall BK and Jones DR: Inhibition of phosphatidylinositol 3-kinase/Akt and histone deacetylase activity induces apoptosis in non-small cell lung cancer in vitro and in vivo. J Thorac Cardiovasc Surg 130: 1422-1429, 2005.
31. Lee HY, Moon H, Chun KH, et al: Effects of insulin-like growth factor binding protein-3 and farnesyltransferase inhibitor SCH66336 on Akt expression and apoptosis in non-small-cell lung cancer cells. J Natl Cancer Inst 96: 1536-1548, 2004.

32. Chang YS, Gong K, Sun S, et al: Clinical significance of insulin-like growth factor-binding protein-3 expression in stage I non-small cell lung cancer. Clin Cancer Res 8: 3796-3802, 2002.

33. Tanaka R, Ishiyama $\mathrm{T}$, Uchihara $\mathrm{T}$, et al: Expression of the Bax inhibitor-1 gene in pulmonary adenocarcinoma. Cancer 106: 648-653, 2002.

34. Lee HY, Chun KH, Liu B, et al: Insulin-like growth factor binding protein-3 inhibits the growth of non-small cell lung cancer. Cancer Res 62: 3530-3537, 2002. 